\title{
Optimization of Nanoparticle-Based SERS Substrates through Large- Scale Realistic Simulations
}

\author{
Diego M. Solís, ${ }^{\dagger}$ José M. Taboada, $^{\ddagger}$ Fernando Obelleiro, ${ }^{* \dagger}{ }^{\dagger}$ Luis M. Liz-Marzán, ${ }^{\S, \|, \text {,I }}$
}

and F. Javier García de Abajo*, ${ }^{*, \#}$

†Departamento de Teoría de la Señal y Comunicaciones, University of Vigo, 36301 Vigo, Spain

${ }^{\ddagger}$ Departamento de Tecnología de Computadores y Comunicaciones, University of Extremadura, 10003 Cáceres, Spain

${ }^{\S}$ Bionanoplasmonics Laboratory, CIC biomaGUNE, Paseo de Miramón 182, 20014 Donostia-San Sebastian, Spain

${ }^{\perp}$ ICFO-Institut de Ciencies Fotoniques, The Barcelona Institute of Science and Technology, 08860 Castelldefels (Barcelona), Spain

"Ikerbasque, Basque Foundation for Science, 48013 Bilbao, Spain

${ }^{\text {II } C I B E R}$ de Bioingeniería, Biomateriales y Nanomedicina, CIBER-BBN, 20014 Donostia-San Sebastian, Spain

\#ICREA-Institució Catalana de Recerca i Estudis Avançats, Passeig Lluís Companys 23, 08010 Barcelona, Spain

Supporting Information

ABSTRACT: Surface-enhanced Raman scattering (SERS) has become a widely used spectroscopic technique for chemical identification, providing unbeaten sensitivity down to the singlemolecule level. The amplification of the optical near field produced by collective electron excitations - plasmons- in nanostructured metal surfaces gives rise to a dramatic increase by many orders of magnitude in the Raman scattering intensities from neighboring molecules. This effect strongly depends on the detailed geometry and composition of the plasmonsupporting metallic structures. However, the search for optimized SERS substrates has largely relied on empirical data, due in part to the complexity of the structures, whose simulation becomes prohibitively demanding. In this work, we use state-of-the-art electromagnetic computation techniques to produce predictive simulations for a wide range of nanoparticle-based SERS substrates, including realistic configurations consisting of random arrangements of hundreds of nanoparticles with various morphologies. This allows us to derive rules of thumb for the influence of particle anisotropy and substrate coverage on the obtained SERS enhancement and optimum spectral ranges of operation. Our results provide a solid background to understand and design optimized SERS substrates.

KEYWORDS: surface-enhanced Raman scattering (SERS), optical sensing, gold nanoparticles, plasmons, particle arrays

$\mathrm{T}^{\mathrm{s}}$ he ability of plasmons to enhance the electric field of light by several orders of magnitude near the surface of metallic nanostructures has found important applications in areas such as optical sensing, ${ }^{1-9}$ photochemistry, ${ }^{10-14}$ and nanomedicine. ${ }^{15-17}$ In particular, a widely used sensing strategy relies on the dependence of the plasmon frequencies on the dielectric environment, which is altered by the presence of the target molecules, revealed through measurable shifts in the spectral positions of the plasmons. ${ }^{18,19}$ Analyte-driven modifications of the nonlinear response of plasmon-supporting structures offer an alternative approach to sensing that has been recently explored as well. ${ }^{20-23}$ Unfortunately, these techniques require the use of specific molecular receptors to enable chemical identification of selectively attached analytes. Chemical identification is also possible through Raman scattering, whose inelastic light signal exhibits spectral features that define molecule-specific barcodes. However, Raman scattering is an extremely inefficient process, so an enhancement mechanism is needed to make it practical. Plasmons provide that mechanism in the so-called surface-enhanced Raman scattering (SERS), which has been demonstrated to reach single-molecule sensitivity when the analyte is placed at narrow gaps between noble metal nanoparticles. ${ }^{1-3,6}$

Although it is well known that several factors affect the interaction of light with molecules in the neighborhood of plasmonic substrates, ${ }^{24-26}$ it is now widely accepted that the main source of enhancement of Raman scattering is driven by the large field amplification generated by plasmons, both on the externally incident light and on the inelastically scattered signal. $^{3,6,26}$ For this reason, much of the activity in the field is directed toward the design of highly efficient SERS substrates,

Received: October 14, 2016

Published: December 20, 2016 
(a)
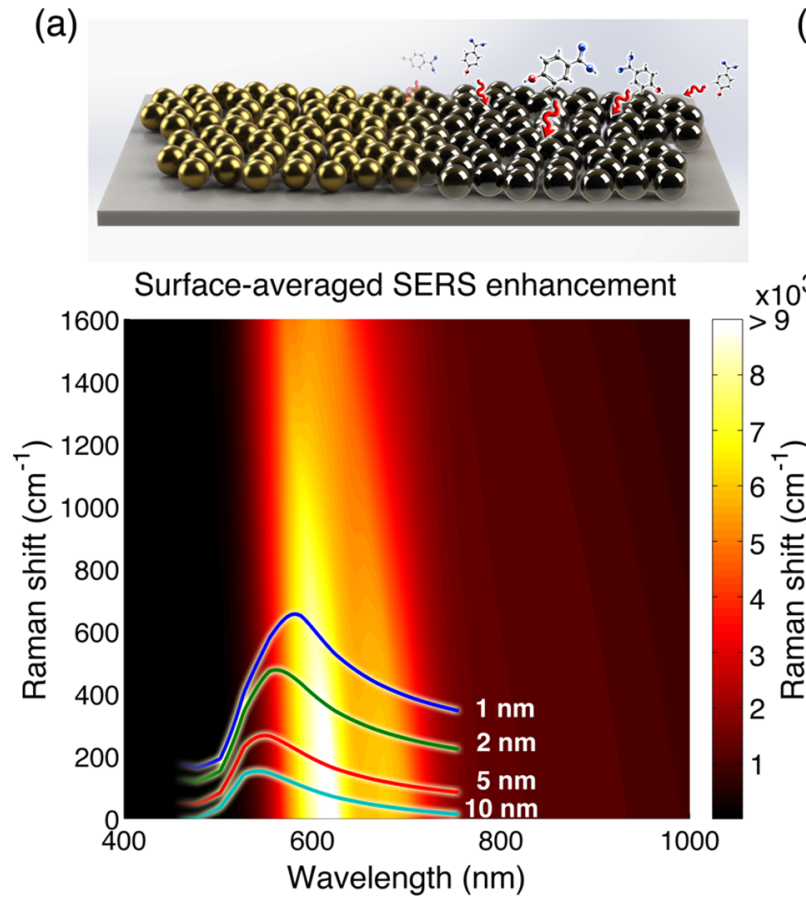

(b)

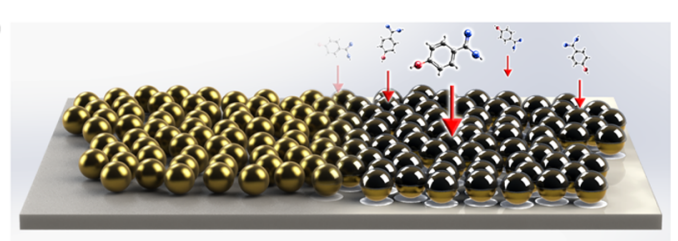

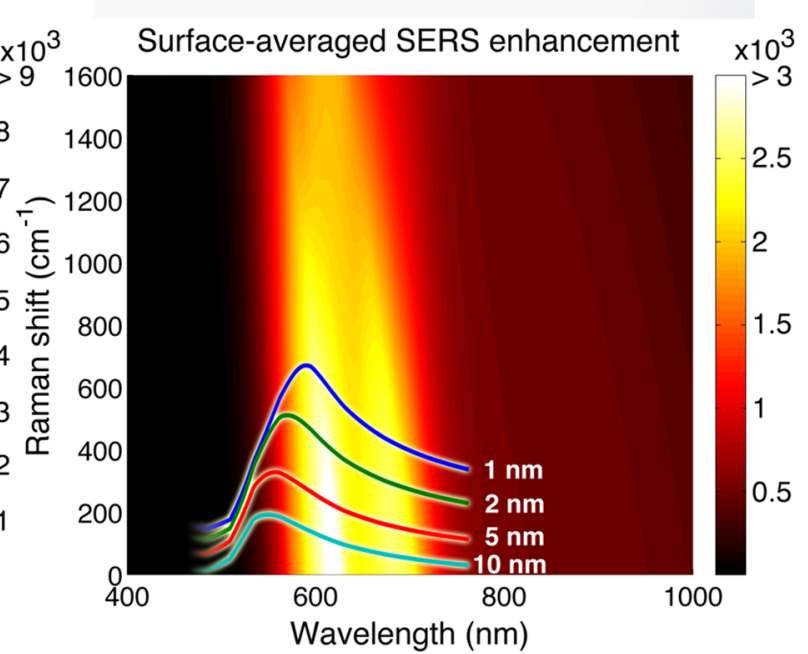

Figure 1. SERS enhancement for two different types of analyte distributions. We consider either skin-type (a) or sheet-type (b) molecular coverages on a randomly arranged monolayer of gold nanospheres (GNPs) deposited on glass. The upper panels show sketches of the geometry and analyte distributions, while the lower panels represent the SERS enhancement averaged over the molecules as a function of light wavelength and Raman shift. In skin-type coverage (a), molecules are distributed with uniform areal density over the gold and glass surfaces, a situation that corresponds to maximum molecular mobility in the interstitial regions before surface attachment. In sheet-type coverage (b), a uniform distribution of molecules is initially assumed on a plane above and parallel to the glass substrate; molecules are then projected downward and fixed at the first surface that they encounter, leaving undecorated regions under the spheres, as well as a nonuniform molecular distribution on the upper spherical surfaces; this corresponds to the limit of minimum molecular mobility. The molecules are considered to be $1 \mathrm{~nm}$ away from the surface in all cases. The simulated arrays consist of 437 GNPs (51 nm diameter) distributed over a $1.2 \times 1.2 \mu \mathrm{m}^{2}$ area, with a minimum surface-to-surface separation of $1 \mathrm{~nm}$ and air above the structure. The SERS intensity is estimated from the incidence-polarization-averaged product of near-field intensities at the incident and inelastically scattered light wavelengths, both calculated under normal irradiation (see Methods). As a reference, we show the SERS enhancement of particle dimers (curves superimposed to color plots) for different gap distances (see labels).

which are pushed to yield increasingly low limits of detection. The composition, size, and morphology of the plasmonic metallic nanostructures configure a large range of possible optimization parameters. By and large, SERS substrate design is currently guided by intuitive application of a few generally accepted rules; namely, silver produces the largest enhancement among the plasmonic materials, although gold is more versatile in practice because of its higher chemical stability and wider variety of available nanoparticle shapes. Additionally, anisotropic morphologies are advantageous to achieve larger field amplification and in particular those that feature sharp tips and edges. Finally, the most efficient hotspots are generated at the gaps between curved metallic surfaces, particularly when the separation is decreased down to $\sim 1 \mathrm{~nm}$.,6,27

Plasmon-driven SERS intensities can be simulated as the product of enhancements due to both the incident light intensity at the position of the molecule and the far-field emission intensity due to the inelastically scattered light. These quantities are in turn obtained by solving the Maxwell equations, which yield the near field produced upon external illumination and the far field driven by the Raman emission dipole at the molecule position. Unfortunately, such electromagnetic simulations require extremely demanding computations for realistic structures and exceed the capability of currently available software packages, whose application is typically restricted to relatively simple geometries, consisting of only a few elements such as spheres, nanorods, and tips. $6,27,28$
Therefore, the interpretation of experimental results is often based on intuitive extrapolations of simulations carried out for very small substrates, which are not necessarily valid because they assume oversimplifications of the actual experimental structures. Nevertheless, it is possible to rely on massive parallelization and advanced surface-integral techniques, combined with heuristic acceleration strategies, to model large, complex plasmonic systems, as we have recently shown with calculations of the electric field and SERS enhancement near multilayers of $>1000$ gold nanorods arranged either randomly or in perfectly ordered supercrystals. ${ }^{29}$ A more detailed analysis of the latter has been recently reported and compared with experimental data. ${ }^{30}$

In this work we aim at a detailed analysis of a widely used configuration of SERS substrates: submonolayers of nanoparticles obtained by drop-casting a colloidal solution on a solid surface, such as a glass slide. The nanoparticles are thus randomly distributed with varying density depending on the colloid concentration and other parameters related to adsorption on the particle surface. We focus on gold particles (the most common plasmonic material) with preferred morphologies for SERS (spheres, rods, and stars) and study the efficiency of SERS substrates when varying the particle coverage within the submonolayer regime, in such a way that the simulated systems closely resemble the experiment. 


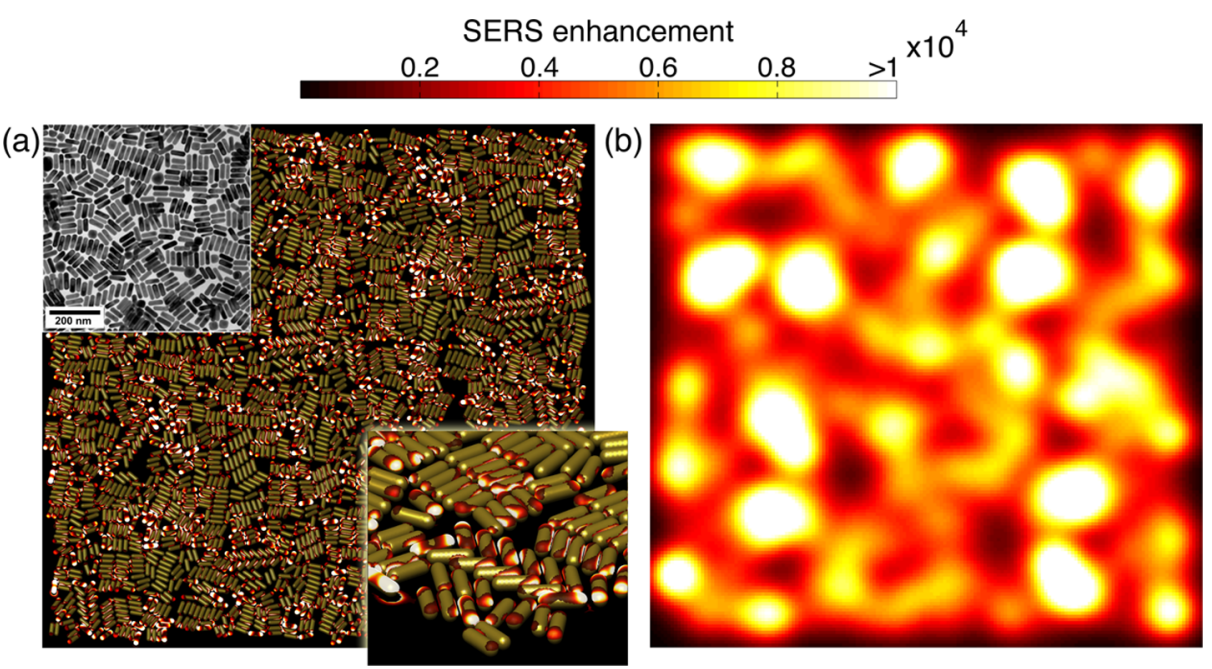

Figure 2. Realistic simulation of SERS enhancement in a nanorod monolayer. (a) SERS intensity map in a planar monolayer of 2930 randomly arranged gold nanorods (GNRs, $65 \mathrm{~nm}$ length, $21 \mathrm{~nm}$ diameter, semispherical caps) spanning an area of $2.4 \times 2.4 \mu \mathrm{m}^{2}$ with a minimum surface-tosurface separation of $1 \mathrm{~nm}$. Top inset: TEM image of an experimental sample. Bottom inset: detail of the simulated geometry showing the distribution of SERS intensities. (b) SERS intensity optical-microscope image obtained from (a) by convoluting with a 2D Gaussian point function ( $0.15 \lambda$ standard deviation, corresponding to a NA of 1.4). The SERS intensity is averaged over incidence light polarizations under normal irradiation at $\lambda_{0}=785 \mathrm{~nm}$ wavelength for zero Raman shift. Skin-type molecular coverage is assumed with $1 \mathrm{~nm}$ surface-molecule separation and a surrounding homogeneous medium of permittivity $\epsilon=1.77$ (water).

\section{RESULTS AND DISCUSSION}

It is important to realize that the actual distribution of analyte molecules is strongly dependent on both the method used to deposit them on the SERS substrate (evaporation, sublimation, microfluidics, etc.) and the adhesion characteristics for each combination of molecule and surface material. Here we consider two extreme situations of molecular distributions, roughly corresponding to the limits of high and small molecular mobilities in a surrounding fluid before attachment: skin- and sheet-type distributions, respectively (Figure 1 top panels). In skin-type coverage (Figure 1a), the molecules have a uniform distribution on both the gold-particles and the glass-substrate surface, which is consistent with a situation in which they can easily penetrate all interstitial regions of the system. In contrast, in sheet-type coverage (Figure 1b) we consider deposition following a restricted downward molecular diffusion along the substrate normal, assuming a uniform density of molecules per unit of projected area (i.e., starting with a uniform planar sheet of molecules parallel to the substrate), which obviously leads to nonuniform molecular distributions on the curved surfaces of the particles, as well as a depletion of molecules at the interparticle gaps.

A simulation of the average SERS enhancement (see Methods) is presented in Figure 1 (lower panels) for these two extreme cases of molecular coverage, using a substrate consisting of $437 \mathrm{Au}$ nanospheres (GNPs, $51 \mathrm{~nm}$ diamater) randomly deposited on a $1.2 \times 1.2 \mu \mathrm{m}^{2}$ area of a glass surface (for comparison, we note that the maximum number of GNPs in a hexagonal close-packed monolayer fitting that area is 479). We limit the minimum surface-to-surface interparticle separation to $1 \mathrm{~nm}$, which is consistent with typical sizes of analyte molecules and capping ligands and is an accepted value of gaps in highly efficient hotspots. Furthermore, we assume the particles to be directly stuck onto the glass surface.

The color plots in Figure 1 show the SERS enhancement, estimated from the product of electric-near-field intensities at the incident and emission wavelengths (see Methods) as a function of excitation wavelength and Raman shift. The plots are dominated by an intense feature near $600 \mathrm{~nm}$ wavelength, due to the amplification of the optical near field produced by interparticle gap plasmons, which are effectively averaged over the distribution of gap distances (see field enhancement spectra superimposed on the color plots for dimers with different gaps). Interestingly, the maximum SERS enhancement in GNP dimers (solid curves in Figure 1) red-shifts with decreasing gap distance but is still lying to the blue with respect to the maximum for the array, as a result of plasmonic modes involving more than two particles. Additionally, the skin coverage leads to $\sim 3$ times higher enhancement, which is expected because in this configuration there is a higher density of analytes in the interparticle gap regions. In both scenarios, the optimum illumination wavelength decreases with increasing Raman shift, as the maximum product of near-field enhancement of the incident and emitted light corresponds to a situation in which their respective wavelengths are placed roughly symmetrically with respect to the plasmon-resonance peak wavelength, so that the blue-shift in the optimum illumination is approximately half of the Raman shift (e.g., a Raman shift of $1600 \mathrm{~cm}^{-1}$ represents a red-shift of $\sim 53 \mathrm{~nm}$ in emission wavelength, consistent with the observed $\sim 25 \mathrm{~nm}$ blue-shift in optimum incident wavelength). Similar conclusions on the Raman-shift and coverage-model dependences are obtained for monolayers of gold nanorods (GNRs) and nanostars (GNSs) (see Figures S1 and S2 in the Supporting Information, SI), although the maximum enhancement occurs at longer wavelengths (see below).

The Raman signal is typically collected through a microscope in actual experiments. The spatial resolution is then limited by diffraction to a fraction of the wavelength, depending on the numerical aperture (NA) of the objective. We include the effects of diffraction phenomenologically in our calculations by convoluting the SERS enhancement maps (computed on a fine grid of $0.02 \mathrm{~nm}$ spacing) with a two-dimensional Gaussianprofile point function. The validity of this procedure is supported by the agreement between the near electric field 
(a)

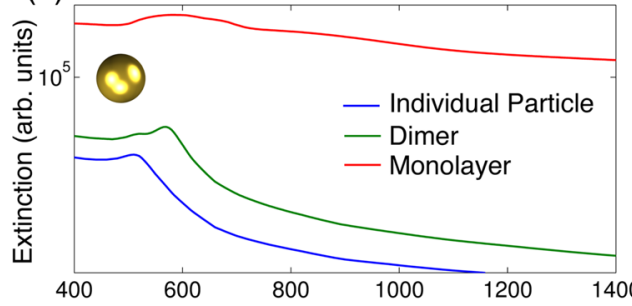

(b)

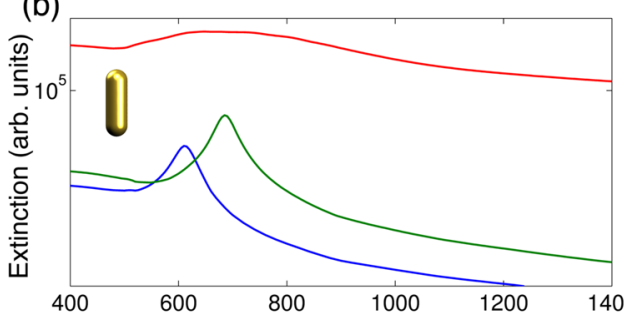

(c)

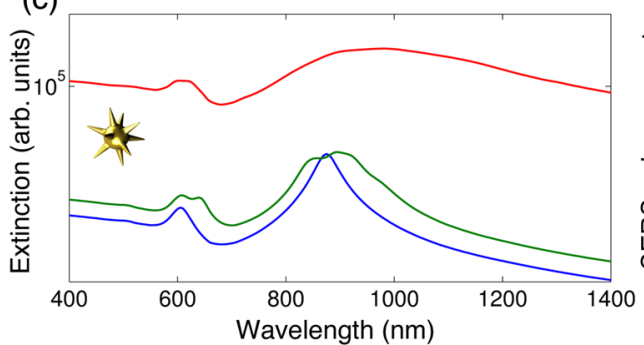

(d)

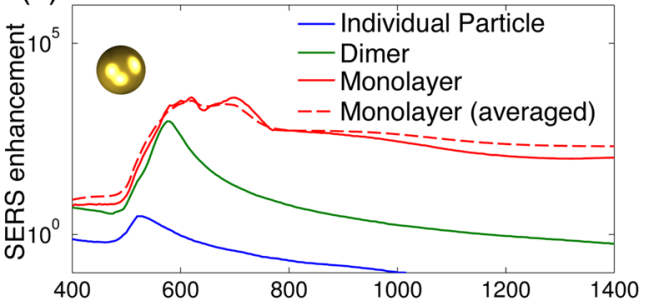

(e)

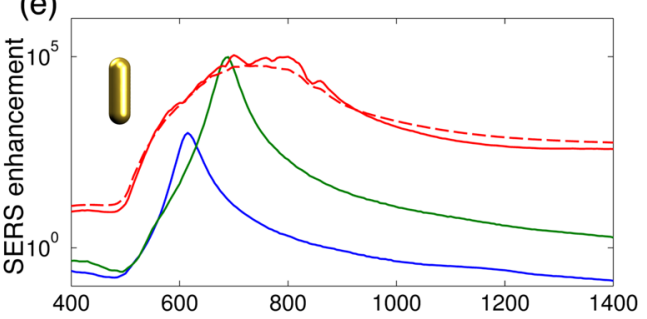

(f)

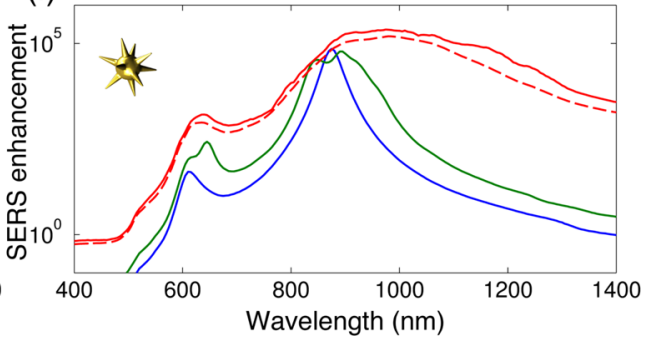

Figure 3. Correlation between optical extinction and SERS enhancement for monomers, dimers, and monolayers of particles with different morphology. $(\mathrm{a}-\mathrm{c})$ Extinction spectra of individual particles, dimers, and randomly arrayed planar monolayers of GNPs (51 nm diameter), GNRs (65 nm length, $21 \mathrm{~nm}$ diameter), and GNSs (core with $20 \mathrm{~nm}$ diameter and 10 branches $15.5 \mathrm{~nm}$ long with tip apexes of $1 \mathrm{~nm}$ ). (d-f) Maximum SERS enhancement observed in the image plane through a NA $=1.4$ objective (see Figure $2 b$ ) for the systems considered in $(a-c)$. The surfaceaveraged SERS enhancement is also shown for the arrays (dashed curves). The monolayers consist of 437 GNPs, 740 GNRs, and 504 GNSs, respectively, randomly arranged on a $1.2 \times 1.2 \mu \mathrm{m}^{2}$ area with $1 \mathrm{~nm}$ minimum gap distance and a homogeneous air environment. All results are averaged over the polarization of the normally incident light for zero Raman shift. Skin-type molecular coverage (see Figure 1) is assumed with a molecule-surface distance of $1 \mathrm{~nm}$.

calculated for an incident Gaussian beam and that obtained under plane-wave illumination after weighing it with a Gaussian profile (see Figure S3 in the SI). Figure 2 illustrates how this transformation of the near-field distribution of SERS enhancements (Figure 2a) leads to a smoother far-field image (Figure 2b). In particular, we consider a monolayer of 2930 GNRs (65 $\mathrm{nm} \times 21 \mathrm{~nm})$ distributed over an area of $2.4 \times 2.4 \mu \mathrm{m}^{2}$ in a random arrangement that mimics actual experiments (cf. measured and calculated geometries in Figure 2a), with a minimum interparticle gap distance of $1 \mathrm{~nm}$. For simplicity, we assume a homogeneous environment of permittivity $\epsilon=1.77$ similar to water. We assume an objective with $\mathrm{NA}=1.4$ by convoluting with a Gaussian of $0.15 \lambda$ standard deviation, where $\lambda$ is the light wavelength in the surrounding medium. ${ }^{31}$ In contrast to the relatively high density of nanoparticles and nearfield optical features, the microscope image displays broad SERS maxima produced by spatial accumulations of hotspots within subwavelength regions.

A cross-comparison of the spectrally resolved optical and SERS performances for the three preferred particle morphologies selected in our work (gold nanospheres, nanorods, and nanostars) is presented in Figure 3. Specifically, we calculate the SERS enhancement as observed in the far field through a NA = 1.4 objective for individual particles, dimers, or monolayers (see further geometrical details in the caption of Figure 3). Plots in the right-hand panels show the maximum of the SERS enhancement in each image (solid curves), while the plots in the left-hand panels represent the optical extinction produced by the same samples. The spectral features of the extinction spectra (Figure $3 \mathrm{a}-\mathrm{c}$ ) are associated with localized surfaceplasmon resonances. Individual GNPs exhibit a characteristic peak near $500 \mathrm{~nm}$ wavelength, while the plasmons of GNRs and GNSs show up at longer wavelengths as a result of the increase in aspect ratio (GNRs) and the presence of sharp tips (GNSs). Retardation also contributes to red-shift the plasmons, but this effect is minor, given the small size of the particles compared with the light wavelength. Aggregation of the particles in dimers and monolayers generally produces additional red-shifts of the spectral features caused by interparticle gaps, as well as an increase in the magnitude of extinction.

Similar conclusions are obtained by analyzing the incidentlight-wavelength dependence of the maximum SERS enhancement observed in the far field (Figure $3 \mathrm{~d}-\mathrm{f}$ ), which increases in magnitude and peaks at longer wavelengths when moving from GNPs to GNRs and GNSs. Additional red-shifts and increase in the magnitude of the SERS enhancement are produced by particle aggregation in dimers and monolayers. This effect is specially important in GNPs and GNRs, in contrast to GNSs. In fact, the presence of sharp tips in the individual GNSs already produces hotspots and ensuing SERS enhancement, while GNS aggregation leads to strong spectral shifts in such hotspots or even quenching when the tips and valleys are closely intertwined, which overall do not add to the shift and 

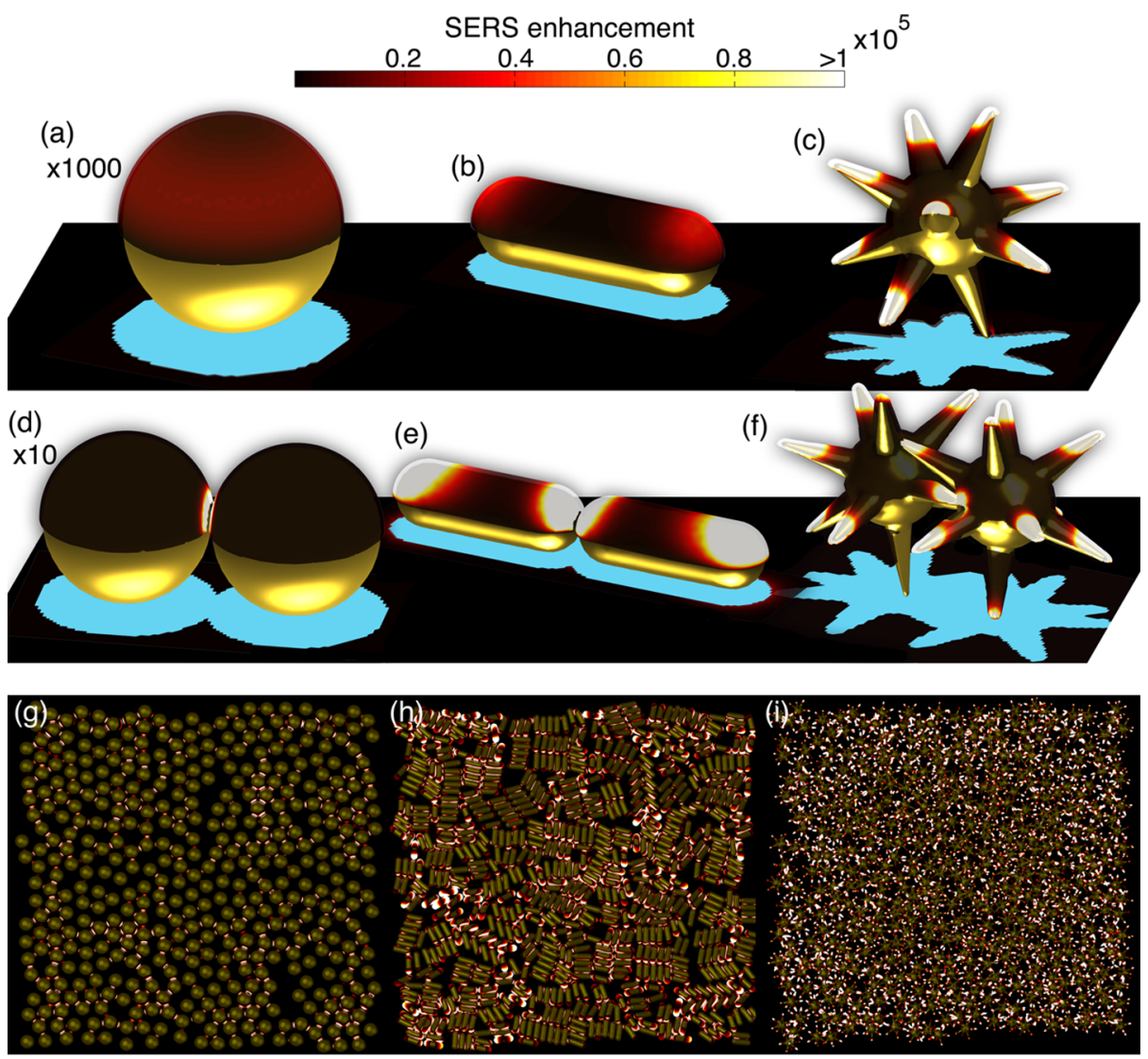

Figure 4. Near-field analysis of SERS enhancement in isolated particles, dimers, and monolayers of GNPs, GNRs, and GNSs. (a-c) Spatial distribution of the SERS enhancement on a molecular skin deposited $1 \mathrm{~nm}$ away from the metal surface (see Figure 1) for individual nanoparticles simulated at the respective incident light wavelengths to yield maximum enhancement for zero Raman shift (525, 615, and $875 \mathrm{~nm}$ for GNPs, GNRs, and GNSs). (d-f) Same as (a)-(c) for dimers (1 nm minimum gap distance), with maxima now shifted to 570, 690, and $890 \mathrm{~nm}$, respectively. (g-i) Same as (d)-(f) for planar monolayers (437 GNPs, 740 GNRs, and 504 GNSs, respectively, randomly distributed on a $1.2 \times 1.2 \mu \mathrm{m}^{2}$ area) calculated at the new peak wavelengths $(620,700$, and $980 \mathrm{~nm}$, respectively). All results are averaged over the polarization of the normally incident light. A homogeneous air environment is assumed. Data in (a) and (d) are multiplied by factors of 1000 and 10, respectively.

increase in Raman signal. Further inspection of the spatially resolved near-field SERS enhancement (Figure 4c,f) corroborates this interpretation, revealing the presence of hotspots that extend form the tips to the central core of the GNSs, while the strength and density of these hotspots are similar both in individual GNSs and in dimers. For monolayers of tightly packed nanoparticles (Figure 3, red curves), we find a good correspondence between the spectral dependences of the optical extinction and the SERS enhancement, which comprise broad maxima resulting from a dense spectral distribution of resonances associated with the varied gap morphologies of randomly occurring gaps.

A systematic analysis of arrays for the three types of particles under consideration is presented in the SI (Figures S4-S6) for molecule-surface separations of 1,2, and $3 \mathrm{~nm}$ and either skinor sheet-type molecular coverage. A reduction in SERS enhancement is observed when the separation is increased, quantified in approximately 2 orders of magnitude lower intensity when moving from 1 to $3 \mathrm{~nm}$. Overall, skin coverage produces larger SERS enhancements, although in the GNS samples they are very close to the values obtained with sheet coverage, a result that we attribute to the dominant role of tips for these particles, which are similarly exposed to molecular attachment with both coverage models. Additionally, we find the far-field enhancement to closely follow the surface-averaged near-field SERS intensity in all cases.
We obtain further insight into the origin of this behavior by examining the near-field SERS enhancement for isolated particles, dimers, and monolayers of GNPs, GNRs, and GNSs. In Figure 4 we present enhancement maps calculated at the corresponding peak light wavelengths (see Figure $3 \mathrm{~d}-\mathrm{f}$ ) in each case. These simulations confirm that the SERS efficiency of individual GNPs or GNRs is not particularly high (Figure 4a,b), whereas plasmon coupling leads to strongly confined resonances that act as hotspots in both dimers (Figure $4 \mathrm{~d}, \mathrm{e}$ ) and dense monolayers (Figure $4 \mathrm{~g}, \mathrm{~h}$ ). In contrast, the multiple tips that branch out from the central core in GNSs support plasmons that are strongly confined at the tips, where they produce hotspots with no need for plasmon hybridization (Figure 4c). Despite the wide variety of conformations that are possible for GNS dimers (including some exceptionally efficient ones, such as coplanar tip-to-tip and tip-to-valley arrangements ${ }^{29}$ ), assembly in dense films does not generally lead to an increase in SERS enhancement or in the number of hotspots. Often, GNSs are intertwined with valley-to-tip contacts (Figure 4 f; see also ref 30 for TEM images of experimental samples), which do not generate more efficient hotspots and can even damp those of the individual particles (see below). Hence, the maximum SERS enhancement is similar for individual GNSs, valley-to-valley dimers, and dense monolayers, as shown in Figure $4 c, f, i$. 

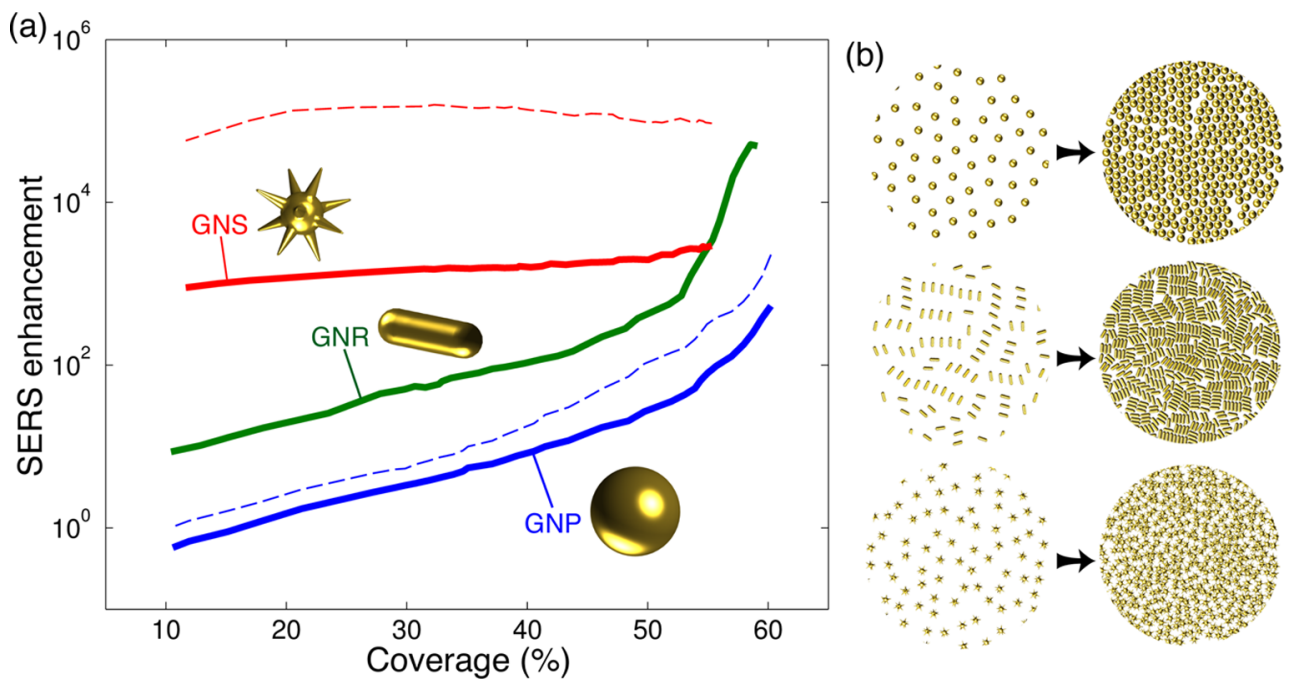

Figure 5. Density dependence of the SERS performance in nanoparticle monolayers. (a) Maximum SERS enhancement as observed through a NA = 1.4 objective (see Figure 2b) for planar monolayers of particles with different morphology (see legend). The particle coverage is defined as the fraction of area occupied by the projection of the metal along the plane normal. (b) Sketches showing an increasing density of nanoparticles in the monolayers. All results are averaged over polarizations of the normally incident light. Solid curves are calculated at $785 \mathrm{~nm}$ incident wavelength for zero Raman shift, while dashed curves for GNPs and GNSs correspond to 633 and $900 \mathrm{~nm}$, respectively. Skin-type molecular coverage (see Figure 1) is assumed with a molecule-surface separation of $1 \mathrm{~nm}$ and a homogeneous air environment.
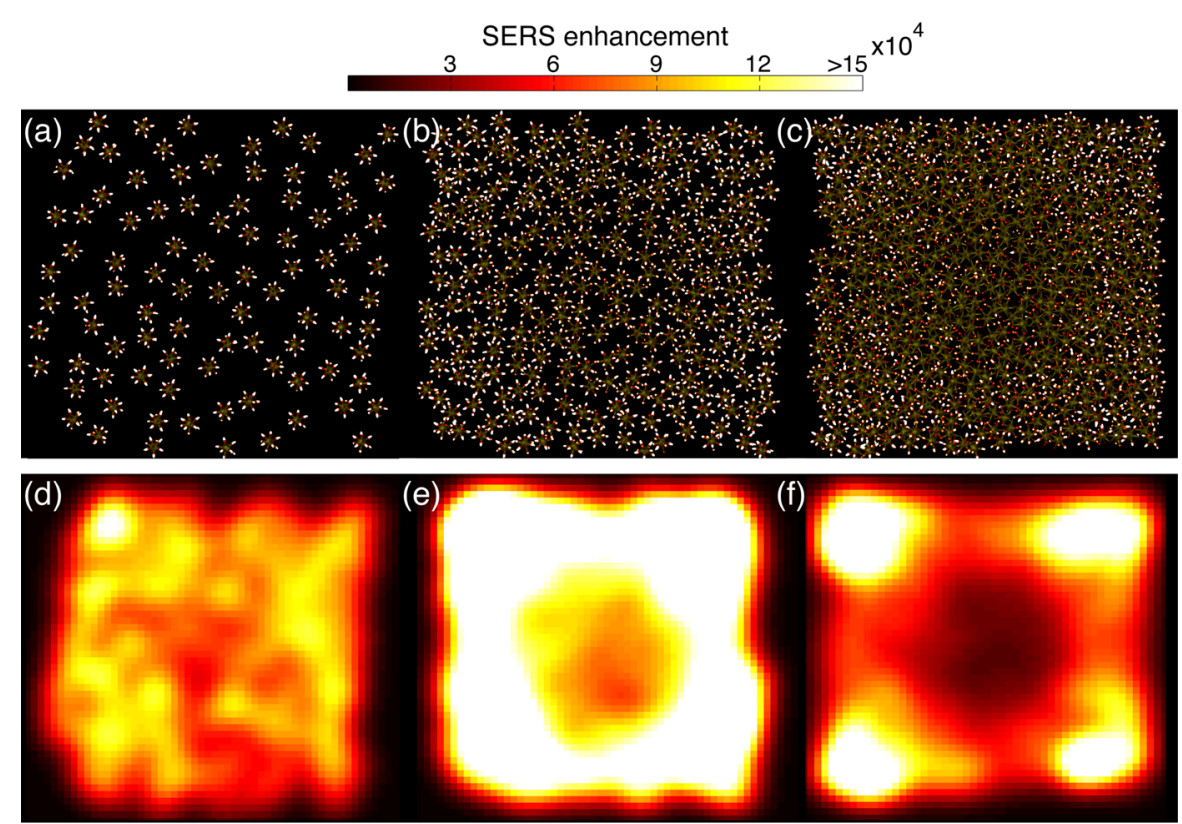

Figure 6. SERS performance of GNS monolayers with different coverages. (a-c) SERS enhancement at $1 \mathrm{~nm}$ distance from the surface of 92 (a), 254 (b), and 504 (c) GNSs randomly distributed on a $1.2 \times 1.2 \mu \mathrm{m}^{2}$ planar area, corresponding to $11.6 \%, 31.4 \%$, and $55.32 \%$ coverage (see Figure 5a), respectively. The SERS intensity is averaged over polarizations of the normally incident light at $900 \mathrm{~nm}$ wavelength for zero Raman shift. A homogeneous air environment is assumed. $(d-f)$ SERS enhancement in the image plane calculated from (a) $-(c)$ for a NA $=1.4$ objective (see Figure $2 b)$.

A striking observation that is particularly evident for GNPs (Figure $4 \mathrm{~g}$ ) is the accumulation of hotspots as a result of the formation of optical standing waves in the particle monolayer. A strong dependence on wavelength and on the overall morphology of the monolayer boundaries confirms the standing-wave nature of these collective modes. Nevertheless, the net contribution to the SERS enhancement remains almost unaffected by the specific distribution of the GNPs in the monolayer island. This effect can also be observed for GNRs (Figure 4h), even though the intrinsic anisotropy of these nanoparticles blurs the formation of standing waves. GNSs present a more complex behavior that we analyze in more detail below.

The particle density in SERS substrates provides a simple and effective parameter for optimization. We investigate the dependence of the SERS performance on particle density for GNPs, GNRs, and GNSs in Figure 5, where the SERS enhancement is plotted as a function of particle coverage. We define the latter as the fraction of surface area occupied by the projection of the metal along the layer plane normal. Solid curves in Figure 5a represent the maximum SERS enhancement as observed in the far field (same near-to-far-field conversion 
procedure as in Figure 3), calculated under normal illumination at $785 \mathrm{~nm}$ (a standard SERS excitation wavelength, in particular for bioapplications) for zero Raman shift. These results reveal a rapid increase in the SERS enhancement with particle density for both GNPs and GNRs (note that the vertical scale is logarithmic), eventually turning into a sudden growth above a coverage of $\sim 50 \%$, followed by saturation around $\sim 60 \%$. The noted turning point is presumably associated with a threshold for more frequent formation of narrow gaps and hotspots with every new added particle. The behavior of GNSs is however rather different: the SERS enhancement is already high in dilute GNS monolayers, and it undergoes only a comparatively slow increase with increasing particle coverage. Interestingly, dense monolayers of GNRs ( $>50 \%$ coverage) exceed the enhancement of GNSs by at least 1 order of magnitude at the highest density under consideration. This picture becomes even more striking when considering laser wavelengths near the optimum performance for each kind of nanoparticle (633 nm for GNPs, $900 \mathrm{~nm}$ for GNSs, dashed curves in Figure 5a): the SERS enhancement is only slightly higher for GNPs, while for GNSs it reaches a maximum at $\sim 30 \%$ coverage and then decreases with increasing particle density (see also Figure S7 in the SI). Incidentally, we note that the enhancement factors that we report here are somewhat lower than those observed experimentally, $^{32}$ which is understandable because we are limiting our calculations to $2 \mathrm{D}$ monolayers, in contrast to the thicker structures used in those measurements, so that the number of hotspots per unit of substrate area is smaller. This effect should not affect our qualitative conclusions.

In an attempt to explain this anomalous behavior of GNSs, we examine SERS enhancement maps calculated for layers with different particle density (Figure 6). Calculations are carried out at $900 \mathrm{~nm}$ incidence light wavelength and zero Raman shift. Inspection of Figure $6 a-c$ reveals an evacuation of hotspots from the central area of the monolayer island as the coverage increases. This behavior is reminiscent of homogeneous plates, where field enhancement takes place mainly at the boundaries. ${ }^{33}$ The far-field images obtained by smoothing the near-field maps (Figure $6 \mathrm{~d}-\mathrm{f}$ ) show the depletion of SERS enhancement in the central region even more clearly. It should be noted that the SERS enhancements represented in Figure 6 are averaged over incident light polarizations. In contrast, when the incident light is linearly polarized along two of the edges of the square-shaped island, an accumulation of SERS enhancement is observed in these two edges (see Figures S8 and S9 in the SI), following a distribution of field enhancement that is typically observed in homogeneous plates. Therefore, we attribute this behavior to the formation of an effective metamaterial associated with the dense arrangement of randomly distributed GNSs, whose central part responds as an effective homogeneous medium, thus preventing the formation of hotspots, which are instead accumulating at the boundaries.

\section{CONCLUSIONS}

In summary, detailed simulations of realistic nanoparticle-based SERS substrates allow us to extract the following general conclusions: (1) particles of higher anisotropy produce maximum performance at longer wavelengths, where they display their intrinsic plasmons; (2) nanostars, which are representative of a high degree of anisotropy, exhibit large enhancement even in the single-particle limit, while aggregation into dense arrays does not lead to significantly better performance; (3) in contrast, nanospheres and nanorods start out with relatively poor SERS efficiencies in dilute layers, but undergo a large boost in the accumulation of hotspots and ensuing SERS efficiency at surface coverages above $\sim 50 \%$, and more precisely, nanorods exceed the SERS enhancement of nanostars at coverages beyond $\sim 60 \%$ at $785 \mathrm{~nm}$ wavelength; (4) nanostars outperform the other two morphologies at their optimum wavelength, which unfortunately occurs more to the red, where the intrinsic Raman cross-section of the molecules is significantly reduced; (5) for nanostars, the formation of relatively homogeneous particle films can be detrimental because they behave as an effective metamaterial, thereby preventing the formation of hotspots. The morphology of the nanoparticles can thus be optimized to produce maximum enhancement for different combinations of laser wavelength and Raman shift. We have covered the most commonly used particle morphologies for SERS, but the calculation procedure would be similar for other shapes. We conclude that, for the commonly used laser wavelength of $785 \mathrm{~nm}$, which offers a compromise between SERS enhancement and intrinsic Raman cross-section, nanorods constitute an excellent option for the fabrication of highly efficient SERS substrates obtained at high particle coverage, outperforming nanospheres by $\sim 2$ orders of magnitude and offering similar and sometimes higher performance than nanostars.

\section{METHODS}

Numerical Solution of Maxwell's Equations. We use a full-wave solution based on surface-integral equations (SIEs) discretized by the method of moments (MoM). ${ }^{29,34,35}$ In SIEMoM, the parametrization and subsequent numerical analysis are both restricted to the two-dimensional boundary surfaces of the particles. This results in a drastic reduction in the number of unknowns compared with other approaches, thus rendering the simulation feasible despite the large size of the systems under consideration. SIE-MoM offers unbeaten accuracy for modeling unbounded electromagnetic scattering problems without the need of absorbing boundary conditions. Additionally, it is robust against rapid oscillations of the fields, and therefore, is particularly suited to deal with narrow gaps such as those encountered in SERS substrates. We further use a multilevel fast multipole algorithm ${ }^{36}$ (MLFMA) combined with the fast Fourier transform ${ }^{37}$ (FFT) for an efficient solution of the dense complex matrix system resulting from SIE-MoM. We also note that MLFMA-FFT provides optimum computational cost and scalability of multiprocessor parallelization. Convergence is dramatically increased by using a multilevel nonoverlapping additive Schwarz domain decomposition preconditioner. ${ }^{38-40}$ Gold and glass are described through their frequency-dependent complex permittivities, taken from optical measurements. ${ }^{41,42}$

Calculation of SERS Enhancement. We simulate the position-dependent SERS enhancement as the product of nearelectric-field enhancements produced upon normal irradiations with light of wavelengths corresponding to the laser light and the inelastically emitted Raman signal, respectively. Several of the results are presented for zero Raman shift, for which the enhancement is simply given by $\left|E / E^{\text {ext }}\right|^{4}$, where $E^{\text {ext }}$ is the incident laser field and $E$ is the resulting near field. This simple definition has the virtue of being independent of the parameters of the optical system used to observe the enhancement (e.g., $\mathrm{NA}$ and acceptance angular ranges). 
Design of SERS Substrates. We assemble nanostars by gluing 10 nanotips (conical shape with $1 \mathrm{~nm}$ apex rounding radius, $15.5 \mathrm{~nm}$ length, and $16.6^{\circ}$ angle) to a spherical core (20 $\mathrm{nm}$ diameter). Monolayers are constructed by iteratively dropping nanoparticles, moving them until they are situated 1 $\mathrm{nm}$ away from the substrate plane, starting from random lateral positions, and discarding particles whose surface is closer than 1 $\mathrm{nm}$ to the surface of any previously deposited particle.

\section{ASSOCIATED CONTENT}

\section{S Supporting Information}

The Supporting Information is available free of charge on the ACS Publications website at DOI: 10.1021/acsphotonics.6b00786.

Additional calculations similar to those in Figure 1 for GNRs and GNSs; an analysis of the dependence on molecule-surface separation and coverage model that supplements the results of Figure 3; an extension of Figure 5 to consider different incidence wavelengths and molecule-surface separations; and a study of the lightpolarization dependence of the SERS performance in the arrays of Figure 6 (PDF)

\section{AUTHOR INFORMATION}

\section{Corresponding Authors}

*E-mail (F. Obelleiro): obi@com.uvigo.es.

*E-mail (F. J. García de Abajo): javier.garciadeabajo@icfo.es.

\section{Notes}

The authors declare no competing financial interest.

\section{ACKNOWLEDGMENTS}

This work was supported in part by the European Regional Development Fund (ERDF), the Spanish MINECO (MAT2014-58201-C2-1-R, MAT2014-58201-C2-2-R, MAT2014-59096-P, MAT2013-46101-R, and SEV2015-0522), the Galician (Atlantic Research Center for Information and Communication Technologies, AtlantTIC) and Extremadura (IB13185) Regional Governments, the European Commission (CNECT-ICT-604391 and FP7-ICT- 2013-613024-GRASP), and Fundació Privada Cellex. L.M.L.-M. acknowledges funding from ERC (Advanced Grant No. 267867 Plasmaquo).

\section{REFERENCES}

(1) Kneipp, K.; Wang, Y.; Kneipp, H.; Perelman, L. T.; Itzkan, I.; Dasari, R. R.; Feld, M. S. Single molecule detection using surfaceenhanced Raman scattering (SERS). Phys. Rev. Lett. 1997, 78, 16671670.

(2) Nie, S.; Emory, S. R. Probing single molecules and single nanoparticles by surface-enhanced Raman scattering. Science 1997, 275, 1102-1106.

(3) Xu, H.; Bjerneld, E. J.; Käll, M.; Börjesson, L. Spectroscopy of single hemoglobin molecules by surface enhanced Raman scattering. Phys. Rev. Lett. 1999, 83, 4357-4360.

(4) Moskovits, M. Surface-enhanced Raman spectroscopy: a brief retrospective. J. Raman Spectrosc. 2005, 36, 485-496.

(5) Anker, J. N.; Hall, W. P.; Lyandres, O.; Shah, N. C.; Zhao, J.; Van Duyne, R. P. Biosensing with plasmonic nanosensors. Nat. Mater. 2008, 7, 442-453.

(6) Rodríguez-Lorenzo, L.; Álvarez-Puebla, R. A.; Pastoriza-Santos, I.; Mazzucco, S.; Stéphan, O.; Kociak, M.; Liz-Marzán, L. M.; García de Abajo, F. J. Zeptomol detection through controlled ultrasensitive surface-enhanced Raman scattering. J. Am. Chem. Soc. 2009, 131, 4616-4618.
(7) Lu, G.; De Keersmaecker, H.; Su, L.; Kenens, B.; Rocha, S.; Fron, E.; Chen, C.; Van Dorpe, P.; Mizuno, H.; Hofkens, J.; et al. Live-cell SERS endoscopy using plasmonic nanowire waveguides. Adv. Mater. 2014, 26, 5124-5128.

(8) Pazos-Perez, N.; Pazos, E.; Catala, C.; Mir-Simon, B.; Gómez de Pedro, S.; Sagales, J.; Villanueva, C.; Vila, J.; Soriano, A.; García de Abajo, F. J.; et al. Ultrasensitive multiplex optical quantification of bacteria in large samples of biofluids. Sci. Rep. 2016, 6, 29014.

(9) Bodelón, G.; Montes-García, V.; López-Puente, V.; Hill, E. H.; Hamon, C.; Sanz-Ortiz, M. N.; Rodal-Cedeira, S.; Costas, C.; Celiksoy, S.; Pérez-Juste, I. Detection and imaging of quorum sensing in Pseudomonas aeruginosa biofilm communities by surface-enhanced resonance Raman scattering. Nat. Mater. 2016, 15, 1203-1211.

(10) Mukherjee, S.; Libisch, F.; Large, N.; Neumann, O.; Brown, L. V.; Cheng, J.; Lassiter, J. B.; Carter, E. A.; Nordlander, P.; Halas, N. J. Hot Electrons Do the Impossible: Plasmon-Induced Dissociation of $\mathrm{H}_{2}$ on Au. Nano Lett. 2013, 13, 240-247.

(11) Baffou, G.; Quidant, R. Nanoplasmonics for Chemistry. Chem. Soc. Rev. 2014, 43, 3898-390710.1039/c3cs60364d.

(12) Clavero, C. Plasmon-Induced Hot-Electron Generation at Nanoparticle/Metal-Oxide Interfaces for Photovoltaic and Photocatalytic Devices. Nat. Photonics 2014, 8, 95-103.

(13) Park, J. Y.; Kim, S. M.; Lee, H.; Naik, B. Hot Electron and Surface Plasmon-Driven Catalytic Reaction in Metal-Semiconductor Nanostructures. Catal. Lett. 2014, 144, 1996-2004.

(14) Moskovits, M. The case for plasmon-derived hot carrier devices. Nat. Nanotechnol. 2015, 10, 6.

(15) Jain, P. K.; Huang, X. H.; El-Sayed, I. H.; El-Sayed, M. A. Noble metals on the nanoscale: Optical and photothermal properties and some applications in imaging, sensing, biology, and medicine. Acc. Chem. Res. 2008, 41, 578-1586.

(16) Qian, X.; Peng, X.-H.; Ansari, D. O.; Yin-Goen, Q.; Chen, G. Z.; Shin, D. M.; Yang, L.; Young, A. N.; Wang, M. D.; Nie, S. In vivo tumor targeting and spectroscopic detection with surface-enhanced Raman nanoparticle tags. Nat. Biotechnol. 2008, 26, 83-90.

(17) Luo, Y. L.; Shiao, Y. S.; Huang, Y. F. Release of photoactivatable drugs from plasmonic nanoparticles for targeted cancer therapy. ACS Nano 2011, 5, 7796-7804.

(18) Liedberg, B.; Nylander, C.; Lunström, I. Surface plasmon resonance for gas detection and biosensing. Sens. Actuators 1983, 4, 299-304.

(19) Zeng, S.; Baillargeat, D.; Hod, H.-P.; Yong, K.-T. Nanomaterials enhanced surface plasmon resonance for biological and chemical sensing applications. Chem. Soc. Rev. 2014, 43, 3426-3452.

(20) Kim, Y.; Johnson, R. C.; Hupp, J. T. Gold nanoparticle-based sensing of spectroscopically silent heavy metal ions. Nano Lett. 2001, $1,165-167$.

(21) Singh, A. K.; Senapati, D.; Wang, S.; Griffin, J.; Neely, A.; Candice, P.; Naylor, K. M.; Varisli, B.; Kalluri, J. R.; Ray, P. C. Gold nanorod based selective identification of Escherichia coli bacteria using two-photon Rayleigh scattering spectroscopy. ACS Nano 2009, 3, 1906-1912.

(22) Mesch, M.; Metzger, B.; Hentschel, M.; Giessen, H. Nonlinear Plasmonic Sensing. Nano Lett. 2016, 16, 3155-3159.

(23) Yu, R.; Cox, J. D.; García de Abajo, F. J. Nonlinear plasmonic sensing with nanographene. Phys. Rev. Lett. 2016, 117, 123904.

(24) Moskovits, M.; Suh, J. S. Surface selection rules for surfaceenhanced Raman spectroscopy: calculations and application to the surface-enhanced Raman spectrum of phthalazine on silver. J. Phys. Chem. 1984, 88, 5526-5530.

(25) Smith, E.; Dent, G. Modern Raman Spectroscopy: A Practical Approach; Wiley: New York, 2005.

(26) Lombardi, J. R.; Birke, R. L. A unified approach to surfaceenhanced Raman spectroscopy. J. Phys. Chem. C 2008, 112, 56055617.

(27) Li, K. R.; Stockman, M. I.; Bergman, D. J. Self-similar chain of metal nanospheres as an efficient nanolens. Phys. Rev. Lett. 2003, 91, 227402 . 
(28) Pazos-Perez, N.; Wagner, C. S.; Romo-Herrera, J. M.; LizMarzán, L. M.; García de Abajo, F. J.; Wittemann, A.; Fery, A.; AlvarezPuebla, R. A. Organized plasmonic clusters with high coordination number and extraordinary enhancement in surface-enhanced Raman scattering (SERS). Angew. Chem., Int. Ed. 2012, 51, 12688-12693.

(29) Solís, D. M.; Taboada, J. M.; Obelleiro, F.; Liz-Marzán, L. M.; García de Abajo, F. J. Toward ultimate nanoplasmonics modeling. ACS Nano 2014, 8, 7559-7570.

(30) Serrano-Montes, A. B.; Jimenez de Aberasturi, D.; Langer, J.; Giner-Casares, J. J.; Scarabelli, L.; Herrero, A.; Liz-Marzán, L. M. A general method for solvent exchange of plasmonic nanoparticles and self-assembly into SERS-active monolayers. Langmuir 2015, 31, 92059213.

(31) Novotny, L.; Hecht, B. Principles of Nano-Optics; Cambridge University Press: New York, 2006.

(32) Hamon, C.; Novikov, S.; Scarabelli, L.; Basabe-Desmonts, L.; Liz-Marzán, L. M. Hierarchical Self-Assembly of Gold Nanoparticles into Patterned Plasmonic Nanostructures. ACS Nano 2014, 8, 1069410703.

(33) Balanis, C. A. Advanced Engineering Electromagnetics; Wiley: New York, 1989.

(34) Harrington, R. F. Field Computation by Moment Method; IEEE Press: New York, 1993.

(35) Solís, D. M.; Taboada, J. M.; Obelleiro, F. Surface Integral Equation Method of Moments with Multiregion Basis Functions Applied to Plasmonics. IEEE Trans. Antennas Propag. 2015, 63, 21412152 .

(36) Song, J.; Lu, C.-C.; Chew, W. C. Multilevel fast multipole algorithm for electromagnetic scattering by large complex objects. IEEE Trans. Antennas Propag. 1997, 45, 1488-1493.

(37) Taboada, J. M.; Araújo, M. G.; Obelleiro, F.; Rodríguez, J. L.; Landesa, L. MLFMA-FFT parallel algorithm for the solution of extremely large problems in electromagnetics. Proc. IEEE 2013, 101, $350-363$.

(38) Peng, Z.; Lim, K.; Lee, J. F. Non-conformal domain decomposition methods for solving large multiscale electromagnetic scattering problems. Proc. IEEE 2013, 101, 298-319.

(39) Solís, D. M.; Araújo, M. G.; Landesa, L.; García, S.; Taboada, J. M.; Obelleiro, F. MLFMA-MoM For Solving The Scattering Of Densely Packed Plasmonic Nanoparticle Assemblies. IEEE Photonics J. 2015, 7, 4800709 .

(40) Solís, D. M.; Obelleiro, F.; Taboada, J. M. Surface Integral Equation-Domain Decomposition Scheme for Solving Multi-scale Nanoparticle Assemblies with Repetitions. IEEE Photonics J. 2016, 0, 110.1109/JPHOT.2016.2614895.

(41) Johnson, P. B.; Christy, R. W. Optical constants of the noble metals. Phys. Rev. B 1972, 6, 4370-4379.

(42) Palik, E. D. Handbook of Optical Constants of Solids; Academic Press: San Diego, 1985. 